\title{
SI: Non-additive effects of binding site mutations in calmodulin
}

\author{
Sean C. Edington, ${ }^{\dagger, \ddagger}$ D. Brent Halling, "Suzanna M. Bennett, $₫, \S$ Thomas R. Middendorf, \\ Richard W. Aldrich, and Carlos R. Baiz ${ }^{*, \dagger}$ \\ $\dagger$ †epartment of Chemistry, University of Texas at Austin, Austin, TX 78712 \\ $\ddagger$ Current affiliation: Department of Chemistry, Yale University, New Haven, CT 06520 \\ \Department of Neuroscience, University of Texas at Austin, Austin, TX 78712 \\ $\S$ Current affiliation: Department of Molecular 85 Cellular Physiology, Stanford University School of \\ Medicine, Stanford, CA 94305 \\ E-mail: cbaiz@cm.utexas.edu
}




\section{S1. Supplemental Data}

The complete FTIR and 2D IR diagonal slice datasets from which figures in the main text are derived are provided below. For more information about 2D IR data collection and analysis, see our previous publication. ${ }^{1}$

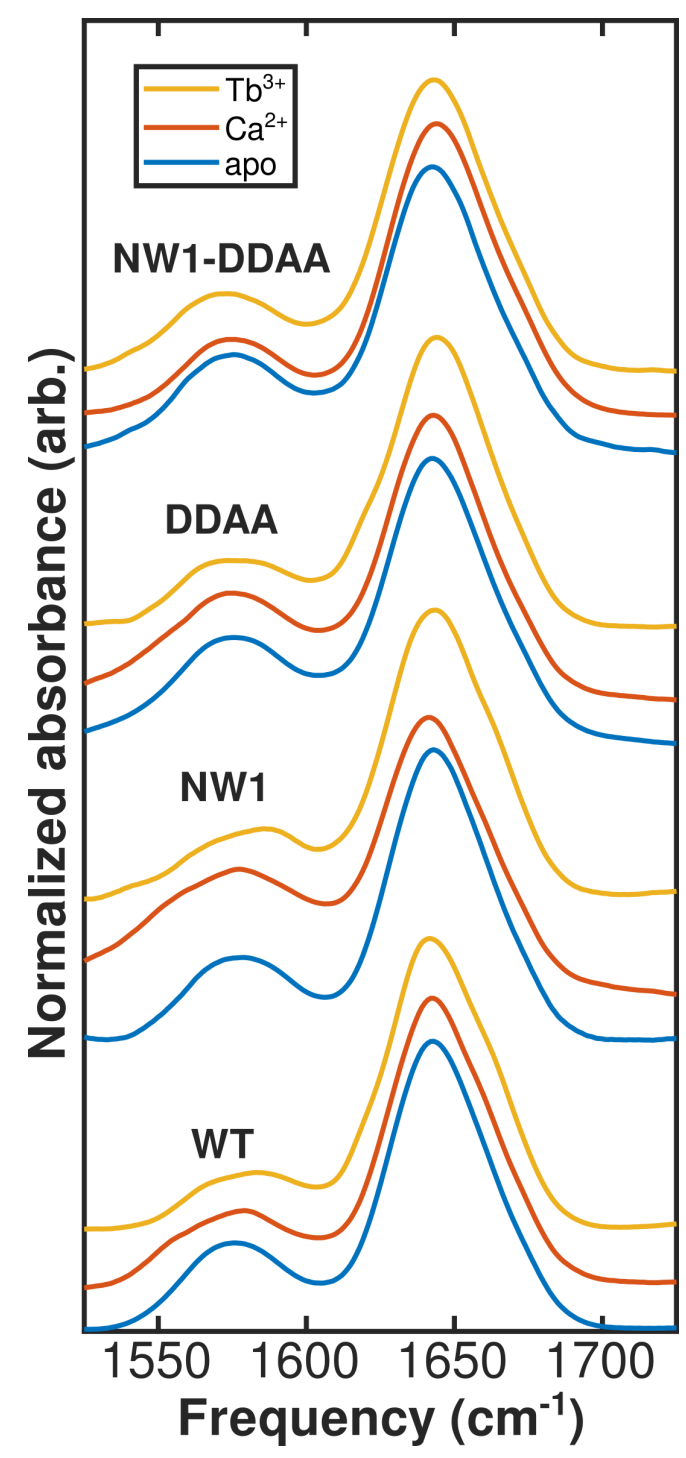

Figure S1: Complete set of FTIR spectra. 


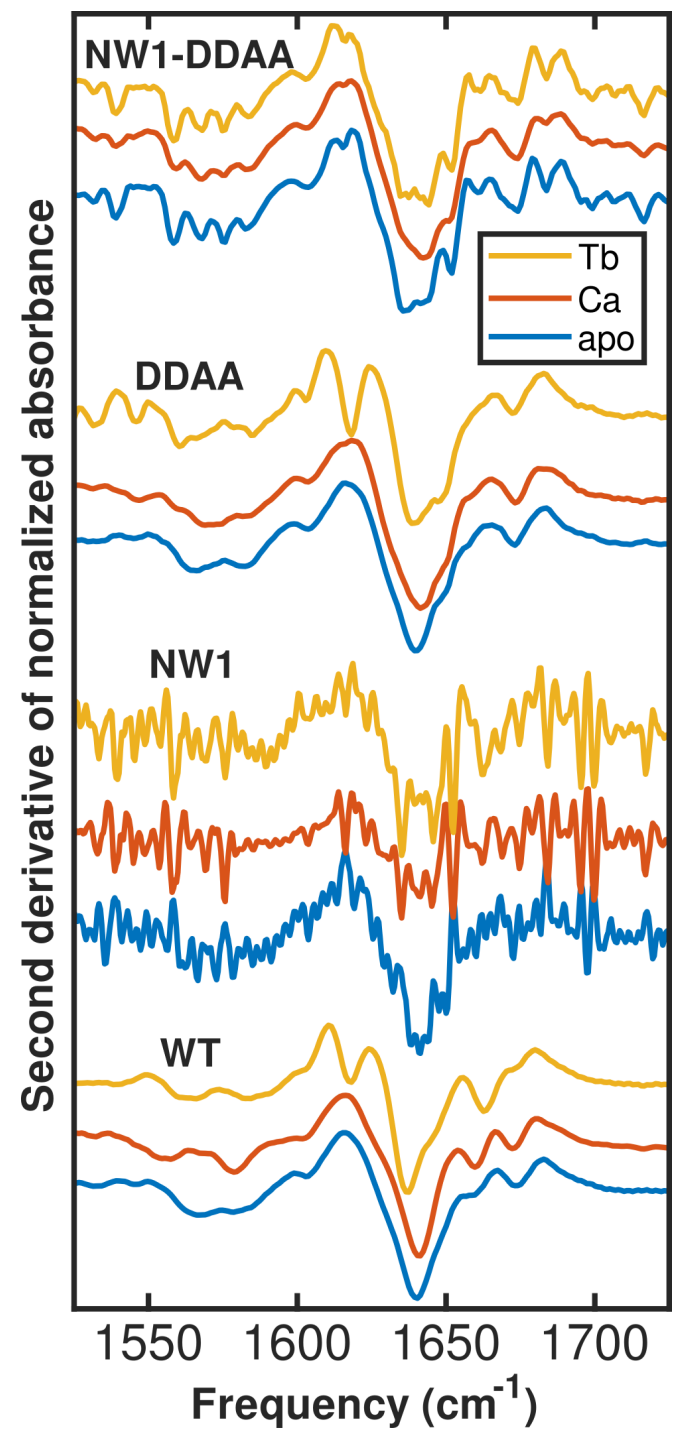

Figure S2: Second derivative spectra for all FTIR spectra shown in Figure S1. While the second derivative spectra show some signatures of carboxylate-mediated ion binding, especially in the WT sample, overall they are less informative than the 2D IR data. Second derivatives for the NW1 spectra show excessive noise because limited availability of the NW1 mutant necessitated use of smaller sample volumes for FTIR experiments, but this limitation does not significantly affect the lineshapes of the raw FTIR spectra. 


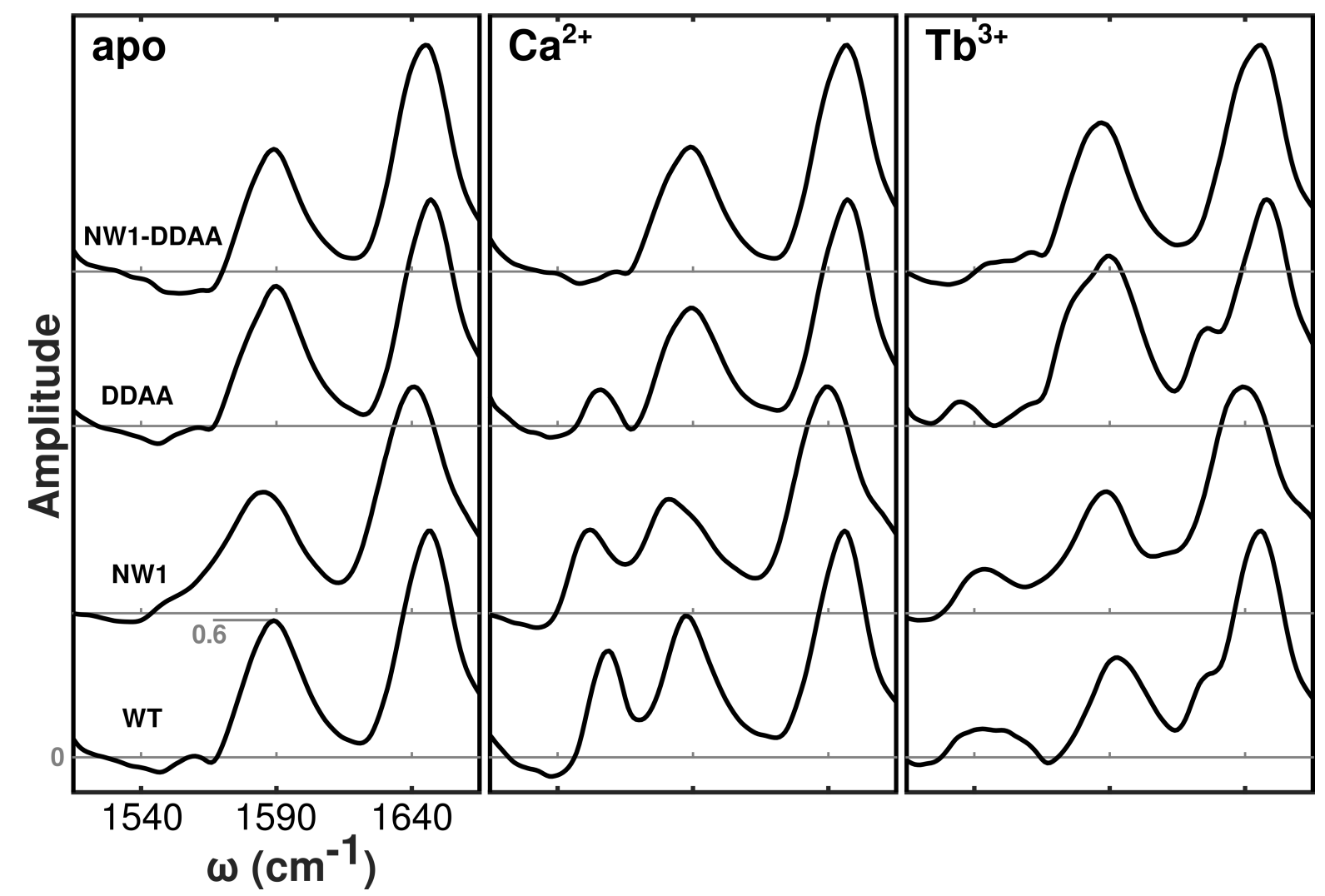

Figure S3: Complete set of 2D IR diagonal traces. Since some of the 2D IR amplitudes are negative, origins for each trace are denoted as grey baselines. 


\section{References}

(1) Edington, S. C.; Gonzalez, A.; Middendorf, T. R.; Halling, D. B.; Aldrich, R. W.; Baiz, C. R. Coordination to lanthanide ions distorts binding site conformation in calmodulin. Proc. Natl. Acad. Sci. U.S.A. 2018, 115, E3126-E3134. 